\title{
Prevalencia de Lesiones de la Mucosa Oral en el Hospital Hernán Henríquez Aravena de Temuco
}

\author{
Prevalence of Oral Mucosal Lesions at the Hernán Henríquez Aravena Hospital of Temuco
}

\author{
"Araceli Raposo; "** María José Monsalves; "***Paola Aravena \& ****Antonio Sanhueza
}

RAPOSO, A.; MONSALVES, M. J.; ARAVENA, P. \& SANHUEZA, A. Prevalencia de lesiones de la mucosa oral en el Hospital Hernán Henriquez Aravena de Temuco. Int. J. Morphol., 29(2):622-627, 2011.

RESUMEN: El propósito de este estudio fue determinar la prevalencia de las lesiones de la mucosa oral en el Hospital Hernán Henríquez Aravena de la ciudad de Temuco, Chile. La muestra fue de 300 pacientes mayores de 20 años, examinados en el mismo hospital (intervalo de confianza del 95\%). La prevalencia de las lesiones de la mucosa oral fue de 66\%. Las lesiones más prevalentes fueron; gránulos de Fordyce (30\%), candidiasis atrófica (14,33\%), mácula melanótica (13,67\%), várices orales (7,33\%), pigmentación fisiológica (6\%), nevos $(4 \%)$, efélides $(3,33 \%)$, úlceras traumáticas $(4 \%)$, leucoplasia (3\%) y queilitis angular $(2,68 \%)$. Se encontró una relación directa entre el aumento de la edad y la presencia de candidiasis atrófica, úlceras traumáticas y várices, siendo esta asociación estadísticamente significativa. Podemos concluir que existe una alta prevalencia de lesiones de la mucosa oral en la población adulta de la muestra y que la cantidad de estas lesiones se incrementan con la edad (candidiasis atrófica, úlceras traumáticas y várices orales), por lo que es necesario intensificar el diagnóstico precoz y el tratamiento oportuno de ellas.

PALABRAS CLAVE: Prevalencia; Epidemiología; Patología oral; Mucosa bucal.

\section{INTRODUCCIÓN}

Las lesiones de la mucosa oral agrupan a un amplio conjunto de alteraciones que se localizan en los tejidos blandos de la cavidad oral, posibles de diferenciar tanto por sus etiologías, características clínicas, pronósticos y tratamientos disímiles.

La prevalencia de las lesiones de la mucosa oral a nivel global ha presentado diferentes valores debido a la diversidad de las muestras en estudio (Andreasen et al., 1986; Campisi \& Margiotta, 2001; Parlak et al., 2006; Mathew et al., 2008). En Chile se presentan escasos estudios, entre los primeros estudios encontramos a Witkop \& Barros (1963) determinó la prevalencia de estas lesiones en población adulta, y el más reciente es el estudio de Espinoza et al. (2006) en el año 2003 que se centró en adultos mayores.

El propósito de este estudio fue conocer la realidad actual de la región de la Araucanía representada por una muestra del Hospital Hernán Henríquez Aravena de Temuco a través de la estimación de la prevalencia de las lesiones de la mucosa oral.

\section{MATERIAL Y MÉTODO}

El presente estudio es de tipo corte transversal. Para la selección del área de estudio, se eligió el Hospital Hernán Henríquez Aravena (H.H.H.A) de la ciudad de Temuco, que es el único establecimiento de mayor complejidad de la red asistencial a la cual pertenece, recibiendo derivaciones de hospitales y consultorios de la región. Se escogió las unidades de servicios de oftalmología, traumatología, otorrinolaringología y módulos del Centro de Diagnóstico y Tratamiento (CDT), con la finalidad de disminuir al máximo la posibilidad de que por su enfermedad de base o por el tratamiento de ésta presentaran como manifestación alguna lesión de la mucosa oral o se estuviese enmascarando alguna lesión preexistente. El universo estuvo constituido por todas las personas que asistieron a las unidades de los servicios antes señalados. Para analizar la distribución de las lesiones pesquisadas, se eliminaron aquellas que no presentaban prevalencia suficiente para los análisis estadísticos, seleccionando así aquellas que permitieron el estudio. Se calculó el número total de la muestra mínimo de sujetos en 289 pacientes asumiendo un intervalo de confianza de $95 \%$, una prevalencia de un $25 \%$ y un margen de error absoluto de un 5\%. Se incluyó a los pacien-

* Cirujano Dentista, Depto. de Cirugía Bucal, Instituto de Odontoestomatología, Universidad Austral de Chile, Chile.

** Cirujano Dentista, Alumna Magíster en Epidemiología Clínica, Universidad de La Frontera, Chile. Asesor Metodológico, Universidad Finis Terrae, Chile.

*** Cirujano Dentista, Departamento de Anatomía Patológica, Facultad de Medicina, Universidad de La Frontera, Chile.

***** Depto. de Matemáticas y Estadística, Facultad de Ingeniería, Ciencias y Administración, Universidad de La Frontera, Chile 
tes de 20 años o más que previamente firmaron el consentimiento informado. Se excluyó a aquellos que no pertenecían a las áreas señaladas, a aquellos que no quisieron ser examinados, a aquellos que presentaron alguna situación particular que les impidió responder la encuesta, o aquellos que no pudieron ser examinados. El protocolo del estudio fue aprobado por los Comités de Ética de la Universidad de la Frontera y del Servicio de Salud Araucanía Sur, Temuco, Chile.

La evaluación de la prevalencia de las lesiones de la mucosa oral fue realizada por un experto en patología oral. Los datos se recogieron en fichas individuales para cada paciente. En las lesiones encontradas que requerían estudio histopatológico se hizo la derivación pertinente a la unidad de servicio maxilofacial para su biopsia, por medio del conducto regular del H.H.H.A. Las lesiones de la mucosa oral estudiadas se determinaron según la Guía de Epidemiología de la OMS (Kramer et al., 1980) y el estudio de Axéll (1976).

Se utilizó una plantilla de recolección de datos para guiar al examinador durante el examen y se grabó en un reproductor de mp3. Los datos fueron traspasados desde la grabación a formato Excel en las plantillas preformadas. Los datos fueron ingresados al programa SAS.V 8.0, con el cual se realizó el análisis estadístico. Se estimaron intervalos de confianza para la prevalencia (95\%) y pruebas de hipótesis para la comparación de las prevalencia en los diferentes grupos $(\mathrm{a}=5 \%)$.

\section{RESULTADOS}

La muestra en estudio correspondió a un total de 300 personas, de las cuales $53,3 \%(\mathrm{n}=160 / 300)$ eran mujeres y $46,67 \%(\mathrm{n}=140 / 300)$ hombres. El promedio de edad de la muestra fue de 46 años. El 43,33\% (n=130/300) era igual o mayor de 20 años y menor o igual de 39 años, el 44,67\% $(n=134 / 300)$ era igual o mayor de 40 años y menor o igual de 69 años y el 12\% (n=36/300) era igual o mayor de 70 años y menor o igual de 89 años.

Del total de la muestra estudiada $(\mathrm{n}=300)$, el $76 \%$ de los pacientes $(n=228 / 300)$ no presentaba enfermedad crónica; el 24\% ( $\mathrm{n}=72 / 300)$ si presentaba enfermedad crónica; el $57 \%(\mathrm{n}=171 / 300)$ no estaba bajo tratamiento medicamentoso, y el 43\% ( $n=129 / 300)$ estaba bajo tratamiento médico. El $27,76 \%$ de los pacientes eran portadores de prótesis dental $(\mathrm{n}=83 / 300)$; mientras que el $72,24 \%$ ( $\mathrm{n}=217 / 300)$ no usaba prótesis dental.

La prevalencia total de lesiones de la mucosa oral en la muestra fue de 66\% ( $\mathrm{n}=198 / 300)$. Su distribución y prevalencia se muestran en la Tabla I.
Tabla I. Prevalencia de lesiones de la mucosa oral en el hospital Hernán Henríquez Aravena, Temuco, Chile.

\begin{tabular}{|c|c|c|}
\hline \multirow{2}{*}{ Lesión } & \multicolumn{2}{|c|}{ Total $(n=300)$} \\
\hline & $\mathbf{n}$ & $\%$ \\
\hline Morsicatio buccarum & 3 & 1 \\
\hline Nevos & 12 & 4 \\
\hline Melanosis por tabaco & 5 & 1,67 \\
\hline Mácula melanótica & 41 & 13,67 \\
\hline Pigmentación fisiológica & 18 & 6 \\
\hline Efélides & 10 & 3,33 \\
\hline Várices & 22 & 7,33 \\
\hline Leucoplasia & 9 & 3 \\
\hline Eritroplasia & 0 & 0 \\
\hline Papiloma & 0 & 0 \\
\hline Gránulos de Fordyce & 90 & 30 \\
\hline Lengua pilosa & 0 & 0 \\
\hline Len gua geográfica & 5 & 1,67 \\
\hline Lengua fisurada & 1 & 0,33 \\
\hline Queilitis actínica & 5 & 1,67 \\
\hline Queilitis angular & 8 & 2,67 \\
\hline C. Eritematosa* & 2 & 0,67 \\
\hline C. Pseudomembranosa* & 0 & 0 \\
\hline Glositis romboidal & 1 & 0,33 \\
\hline C. hiperplásica * & 0 & 0 \\
\hline C. atrófica * & 43 & 14,33 \\
\hline Herpes & 6 & 2 \\
\hline Úlceras traumáticas & 12 & 4 \\
\hline Épulis fisurado & 3 & 1 \\
\hline Fibroma Irritativo & 4 & 1,33 \\
\hline Aftas & 4 & 1,33 \\
\hline
\end{tabular}

*Candidiasis atrófica; Candidiasis hiperplásica; Candidiasis pseudomembranosa; Candidiasis eritematosa.

La presencia de lesiones en mujeres fue de $65,63 \%$ $(\mathrm{n}=105 / 160)$ y en hombres fue de $66,43 \%(\mathrm{n}=93 / 140)$ $(\mathrm{p}=0,88)$. Del total de lesiones pesquisadas, la prevalencia fue de un $47 \%$ ( $n=93 / 198)$ para el sexo masculino y de un 53\% ( $\mathrm{n}=105 / 198)$ para el sexo femenino. En la Tabla II, se muestra la distribución de las lesiones pesquisadas por sexo.

Del grupo de pacientes entre 20 y 39 años $(n=130)$, el 58,46\% ( $\mathrm{n}=76 / 130)$ presentaron lesión; de los pacientes entre 40 y 69 años (n=134), el 69,40\% (n=93/134) presentaron lesión, y del grupo de pacientes entre 70 y 89 años $(\mathrm{n}=36)$, el 80,56\% ( $\mathrm{n}=29 / 36)$ presentaron lesión $(\mathrm{p}=0,024)$. La distribución de lesiones según grupo etario se muestra en la Tabla III. 
Tabla II. Distribución porcentual de lesiones pesquisadas por sexo en el Hospital Hernán Henríquez Aravena, Temuco, Chile.

\begin{tabular}{|c|c|c|c|c|c|c|c|}
\hline \multirow[t]{2}{*}{ Lesión } & \multicolumn{2}{|c|}{ Hombres $(n=140)$} & \multicolumn{2}{|c|}{ Mujeres $(n=160)$} & \multicolumn{2}{|c|}{ Total $(n=300)$} & \multirow[b]{2}{*}{ Valor $p$} \\
\hline & $\mathbf{n}$ & $\%$ & $\mathbf{n}$ & $\%$ & $\mathbf{n}$ & $\%$ & \\
\hline Nevos & 4 & 2,86 & 8 & 5,00 & 12 & 4 & 0,345 \\
\hline Mácula melanótica & 23 & 16,43 & 18 & 11,25 & 41 & 13,67 & 0,193 \\
\hline Pigmentación fisiológica & 7 & 5,00 & 11 & 6,88 & 18 & 6 & 0,495 \\
\hline Efélides & 4 & 2,86 & 6 & 3,75 & 10 & 3,33 & 0,667 \\
\hline Várices & 13 & 9,29 & 9 & 5,63 & 22 & 7,33 & 0,225 \\
\hline Leucoplasia & 4 & 2,86 & 5 & 3,13 & 9 & 3 & 0,892 \\
\hline Gránulos de Fordyce & 47 & 33,57 & 43 & 26,88 & 90 & 30 & 0,207 \\
\hline Queilitis angular & 1 & 0,72 & 7 & 4,38 & 8 & 2,67 & 0,051 \\
\hline C. atrófica $*$ & 17 & 12,14 & 26 & 16,25 & 43 & 14,33 & 0,311 \\
\hline Úlceras traumáticas & 5 & 3,57 & 7 & 4,38 & 12 & 4 & 0,723 \\
\hline
\end{tabular}

*Candidiasis atrófica.

Tabla III. Distribución de lesiones según grupo etario en el Hospital Hernán Henríquez Aravena, Temuco, Chile.

\begin{tabular}{|c|c|c|c|c|c|c|c|c|c|}
\hline \multirow[t]{2}{*}{ Lesión } & \multicolumn{2}{|c|}{$\begin{array}{c}20-39 \\
(n=130)\end{array}$} & \multicolumn{2}{|c|}{$\begin{array}{c}40-69 \\
(n=134)\end{array}$} & \multicolumn{2}{|c|}{$\begin{array}{c}70-89 \\
(n=36)\end{array}$} & \multicolumn{2}{|c|}{$\begin{array}{c}\text { Total } \\
(n=300)\end{array}$} & \multirow[b]{2}{*}{ Valor $p$} \\
\hline & $\mathbf{n}$ & $\%$ & $\mathbf{n}$ & $\%$ & $\mathbf{n}$ & $\%$ & $\mathbf{n}$ & $\%$ & \\
\hline Úlceras traumáticas & 1 & 0,77 & 8 & 5,97 & 3 & 8,33 & 12 & 4 & 0,036 \\
\hline $\mathrm{C}$, atrófica* & 4 & 3,08 & 26 & 19,40 & 13 & 36,11 & 43 & 14,33 & $<0,0001$ \\
\hline Queilitis angular & 1 & 0,77 & 6 & 4,48 & 1 & 2,78 & 8 & 2,67 & 0,17 \\
\hline Gránulos de Fordyce & 36 & 27,69 & 46 & 34,33 & 8 & 22,22 & 90 & 30 & 0,277 \\
\hline Leucoplasia & 5 & 3,85 & 2 & 1,49 & 2 & 5,56 & 9 & 3 & 0,337 \\
\hline Várices & 1 & 0,77 & 11 & 8,21 & 10 & 27,78 & 22 & 7,33 & $<0,0001$ \\
\hline Efélides & 6 & 4,62 & 4 & 2,99 & 0 & 0 & 10 & 3,33 & 0,376 \\
\hline Pigmentación fisiológica & 18 & 13,85 & 0 & 0 & 0 & 0 & 18 & 6 & $<0,0001$ \\
\hline Mácula melanótica & 15 & 11,54 & 20 & 14,93 & 6 & 16,67 & 41 & 13,67 & 0,620 \\
\hline Nevos & 3 & 1,31 & 8 & 5,97 & 1 & 2,78 & 12 & 4 & 0,291 \\
\hline
\end{tabular}

*Candidiasis atrófica.

\section{DISCUSIÓN}

La edad promedio de la muestra nos centra en un grupo etario principalmente adulto joven. La mayoría de los pacientes $(76 \%)$ no presentaba enfermedad crónica; por lo que disminuye la probabilidad de que exista sesgo en la muestra. Un porcentaje no menor de los pacientes (24\%) estaba bajo tratamiento médico, sin embargo, no sería la causante directa de presencia o ausencia de lesiones en la mucosa oral.

Numerosos estudios extranjeros han determinado la prevalencia de lesiones de la mucosa oral, obteniendo diferentes valores asociados a las características propias de cada muestra. La prevalencia determinada a través de nuestro estudio es de un $66 \%$ de lesiones de la mucosa oral. Andreasen et al. (1986) determinó una prevalencia de 25 a $50 \%$ para las lesiones de la mucosa oral; mientras que Parlak et al. obtuvo una prevalencia del $26,2 \%$, Campisi \& Margiotta señaló en su estudio en población italiana una prevalencia de $81,3 \%$ para estas lesiones; un estudio mexicano reportó un 35,6\% de prevalencia (Castellanos \& DíazGuzmán, 2008). Más cercanos a nuestra realidad, encontramos el estudio de Espinoza et al. que arrojó una prevalencia de un 53\%. En comparación con los estudios señalados, encontramos que nuestros resultados se mantienen dentro de 
los rangos anteriomente reportados. Las diferencias encontradas en relación a los estudios de Parlak et al. y Campisi \& Margiotta se justifican esencialmente por las características propias de la muestra. Parlak estudió una población de edad significativamente menor que la muestra de nuestro estudio, mientras que Campisi \& Margiotta investigó a pacientes en una consulta dental; nuestra muestra es más heterogénea en este aspecto, pues los pacientes estudiados no consultaban por este motivo.

La cuantificación de la presencia de lesiones por sexo obtenida en nuestro estudio reveló una relación de 8:10. Estudios extranjeros como el de Castellanos \& Díaz-Guzmán en México, determinó una mayor prevalencia en hombres, con una relación de 1,4:1. Los resultados de nuestro estudio sugieren que el sexo no sería determinante para la presencia de las lesiones estudiadas.

Dentro de este grupo de lesiones encontramos las úlceras aftosas recurrentes orales (URO), con una ocurrencia de $1,53 \%$ en nuestro estudio. Se señala un rango de 5$60 \%$ de presencia de URO en diferentes estudios (Laskin et al., 2003); Katz et al. (2001) señaló una prevalencia de $20 \%$ para estas lesiones; Parlak et al. determinó una prevalencia del 3,6\%; Shulman (2004) encontró un 1-1,4\% de aftas al momento del examen y un 6,9-18,3\% para historia de aftas; Axéll encontró un $2 \%$ de prevalencia de úlceras recurrentes orales (URO) en población adulta; según la OMS (Kramer et al.) la prevalencia de estas lesiones va de un 10-60\%, siendo definida por esta entidad como la lesión oral más frecuente; al comparar estos resultados con nuestro estudio encontramos amplios rangos de diferencias. Esto se debe a las diferentes metodologías entre uno y otro. Así los estudios que presentan mayor porcentaje incluyen también la historia de aftas (Shulman). En nuestra investigación no fue posible determinar la historia de aftas ya que la mayoría de los pacientes no fue capaz de confirmar la presencia de éstas en forma fehaciente a través de su vida, pues las confundían con otras lesiones orales.

Las úlceras traumáticas han sido cuantificadas en un 4\% para el total de nuestra muestra. Reichart (2000) señaló una prevalencia de un $10,1 \%$ en la muestra entre 35 y 44 años, mientras que en la muestra de 65 a 74 años fue de un $1,9 \%$. En ambos grupos la relación entre hombres y mujeres fue de 1:2 aproximadamente. En el estudio de Castellanos \& Díaz-Guzmán, la prevalencia fue de 44,79 por cada 1000 pacientes, siendo la tercera lesión más frecuente. Nuestro estudio presentó un rango muy similar al promedio del total de la muestra observada por Reichart.

La prevalencia de candidiasis en nuestro estudio fue de un $14,33 \%$ en la muestra general. El estudio de Reichart determinó una prevalencia de 2,5\% para el grupo entre 35 a 44 años comparable con el grupo de 20 a 39 años de nuestra muestra que obtuvo una prevalencia de 3,08\%. El estudio de Espinoza consideró una muestra con edades entre 65 a 74 años con una prevalencia de $22,3 \%$; mientras que Reichart señaló una prevalencia de 18,33\% para las mismas edades; comparable con nuestro tercer grupo etario de 70 a 89 años que presentó una prevalencia de $36,11 \%$. Frecuencia que se explicaría por el alto porcentaje de pacientes portadores de prótesis.

Nuestro estudio señaló una prevalencia de queilitis angular de 2,68\% para el total de la muestra. La prevalencia señalada por Reichart es de un 4,5\%. Es posible también comparar el grupo etario de Reichart de 35 a 44 años con una prevalencia de $1,1 \%$ con el grupo de 29 a 39 años de nuestro estudio que arrojó una prevalencia de $0,77 \%$. Espinoza et al. señaló una prevalencia de 2,9\% para las edades entre 65 a 74 años; para el mismo grupo etario Reichart señaló una prevalencia de 3,4\%; comparable con nuestro tercer grupo etario de 70 a 89 años con una prevalencia de $2,78 \%$. Por lo que encontramos amplias similitudes entre estos estudios.

La prevalencia de Gránulos de Fordyce es muy poco referida en los estudios relacionados a lesiones de la mucosa oral, pues muchos autores los consideran como una malformación y no una patología oral. Nuestro estudio mostró una prevalencia de $30 \%$ para la muestra general. Reichart señala una prevalencia de $26,6 \%$ para el grupo de 35 a 44 años y 23,7\% para el grupo de 65 a 74 años; Axéll señaló una prevalencia de $82,8 \%$; en los tres estudios, incluyendo el nuestro, es mayor la presencia de estas lesiones en hombres, pero en nuestro estudio las diferencias entre sexos no fue significativa $(\mathrm{p}=0,206)$.

La prevalencia de leucoplasias en nuestro estudio arrojó una prevalencia del 3\% para la muestra general. En adultos, mayores de 15 años, ha sido reportada en un rango entre $1,1 \%$ en Camboya a 3,6\% en Suecia (Petersen et al., 2005), y un 2,2\% en Turquía (Cebeci et al., 2009). En el estudio de Campisi \& Margiotta se obtuvo un 13,8\% de prevalencia mientras que Espinoza et al. reportaron un 1,7\%; Castellanos \& Díaz-Guzmán determinó que de cada 1000 pacientes un 1,75 presentaba la patología. Otro estudio que abarcó un rango etáreo más amplio en India es el de Mathew et al. que reportó una prevalencia de 1,59\%.

La prevalencia de várices no ha sido claramente determinada. Nuestro estudio determinó una prevalencia general de $7,33 \%$ para estas lesiones y un $27,78 \%$ para el grupo etario entre 69 a 80 años. Rango esperable, principalmente asociado a la tendencia etaria de la muestra (Neville et al., 2002). 
La prevalencia de lesiones pigmentadas de la mucosa oral determinada en nuestro estudio fue de un $28,67 \%$ para el conjunto de estas lesiones; siendo la más prevalente la mácula melanótica con un $13,67 \%$ y la pigmentación fisiológica con un 6\%. El estudio de Espinoza et al. determinó la prevalencia de las lesiones pigmentadas en un 2,8\%; el estudio de Axéll también las agrupa y señala una prevalencia de un $9,94 \%$. Los valores obtenidos en nuestra muestra se asociarían al alto número de población indígena en nuestra región, siendo un factor relevante señalado en los textos de referencia como un antecedente de riesgo para la presencia de algunos tipos de lesiones de este grupo de patologías (Unsal et al., 2001).

Se pudo determinar claramente las lesiones asociadas a la edad como lo muestra la Tabla III, donde es posible determinar que las úlceras traumáticas presentan una mayor prevalencia a medida que la edad aumenta, valor estadísticamente significativo $(\mathrm{p}=0,036)$. Estos valores coinciden con lo señalado por Espinoza et al., quien justifica esta correlación entre úlceras recurrentes orales y edad por la asociación de los pacientes mayores y el uso de prótesis. En el caso de la candidiasis atrófica el patrón de relación se mantiene, al igual que en el anterior, asociado a la edad, con una progresión simétrica asociada al aumento de edad ( $\mathrm{p}=<0,0001)$. Espinoza et al. señalan que la alta prevalencia de estomatitis encontrada en población adulta se asocia principalmente a la falta de limpieza y el uso nocturno de la prótesis. La relación entre candidiasis atrófica y el uso de prótesis en nuestro estudio fue igualmente significativo $(\mathrm{p}=<0,0001)$.

Consecuentemente la relación de esta patología con la edad se debe al uso de prótesis en la población de mayor edad. La queilitis angular no presentó una progresión tan marcada en los rangos etarios establecidos en nuestra investigación. Se presentó con valores similares en el segundo y tercer grupo etario, ambos considerablemente mayores que en el primer grupo, pero no con diferencias significativas entre ellos. Los gránulos de Fordyce $(\mathrm{p}=0,277)$ y las leucoplasias $(\mathrm{p}=0,377)$ no muestran asociación a rango etario, se presentan similares a través de toda la muestra. Por el contrario, las várices presentaron una significativa asociación con la edad ( $\mathrm{p}=<0,0001)$. Textos clásicos de patología oral señalan este punto enfáticamente como una degeneración propia de la edad, donde se pierde tejido conectivo que soporta los vasos (Neville et al.).

Las efélides no presentaron asociación a la edad. La pigmentación fisiológica presentó un valor de asociación altamente significativo en relación a la edad $(\mathrm{p}=<0,0001)$, esto se puede asociar al ciclo de producción de melanina que va aumentando durante la vida, disminuyendo en el adulto mayor (Marimon \& Garrotela, 2003; Ross et al., 2006). La mácula melanótica y los nevos no mostraron asociación a rango etario.

Finalmente podemos concluir que existe una alta prevalencia de lesiones de la mucosa oral en la muestra estudiada. Las lesiones que mostraron mayor asociación a la edad son la candidiasis atrófica, las úlceras traumáticas y las várices. Por estas razones es recomendable seguir la línea de investigación para saber con mayor certeza la prevalencia de estas lesiones a nivel regional y nacional, y así mejorar el diagnóstico precoz por parte de odontólogos generales.

RAPOSO, A.; MONSALVES, M. J.; ARAVENA, P. \& SANHUEZA, A. Prevalence of oral mucosal lesions at the Hernán Henríquez Aravena Hospital of Temuco. Int. J. Morphol., 29(2):622-627, 2011.

SUMMARY: The purpose of this study was to determine the prevalence of oral mucosal lesions at the Hernan Henríquez Aravena Hospital in the city of Temuco Chile. Method: The sample consisted of 300 patients aged over 20 years, examined in the same hospital (confidence interval 95\%). Results: The prevalence of oral mucosal lesions was 66\%. The most prevalent lesions were; Fordyce granules $(30 \%)$, atrophic candidiasis $(14.33 \%)$, oral melanotic macule $(13.67 \%)$, oral mucosal varicosities $(7.33 \%)$, physiological pigmentation $(6 \%)$, nevus $(4 \%)$, ephelis $(3.33 \%)$, traumatic ulcers $(4 \%)$, leukoplakia (3\%) and angular cheilitis $(2.68 \%)$. A direct ratio was found between increased age and the presence of atrophic candidiasis, traumatic ulcers and oral mucoral varicosities, this association being statistically significant. It may be concluded that there is a high prevalence of oral mucosal lesions in the adult population of the sample and that the quantity of lesions increases with age (atrophic candidiasis, traumatic ulcers and mucosal varicosities), making it necessary to intensify their early diagnosis and timely treatment.

KEY WORDS: Prevalence; Epidemiology; Oral pathology; Mouth mucosa.

\section{REFERENCIAS BIBLIOGRAFICAS}

Andreasen, J. O.; Pindborg, J. J.; Hjörting-Hansen, E. \& Axéll, T. Oral health care: more than caries and periodontal disease. A survey of epidemiological studies on oral disease. Int. Dent. J., 36(4):207-14, 1986. 
Axéll, T. A prevalence study of oral mucosal lesions in an adult Swedish population. Odontol. Revy. Suppl., 36:1103, 1976.

Campisi, G. \& Margiotta, V. Oral mucosal lesions and risk habits among men in an Italian study population. $J$. Oral Pathol. Med., 30(1):22-8, 2001.

Castellanos, J. L. \& Díaz-Guzmán, L. Lesions of the oral mucosa: an epidemiological study of 23785 Mexican patients. Oral Surg. Oral Med. Oral Pathol. Oral Radiol. Endod., 105(1):79-85, 2008.

Cebeci, A. R.; Güls sahi, A.; Kamburoglu, K.; Orhan, B. K. \& Oztas, B. Prevalence and distribution of oral mucosal lesions in an adult Turkish population. Med. Oral Patol. Oral Cir. Bucal, 14(6):E272-7, 2009.

Espinoza, I.; Rojas, R.; Aranda, W. \& Gamonal, J. Prevalence of oral mucosal lesions in elderly people in Santiago, Chile. J. Oral Pathol. Med., 32(10):5715,2003 .

Katz, J.; Chaushu, G. \& Peretz, B. Recurrent oral ulcerations associated with recurrent herpes labialis-two distinct entities? Community Dent. Oral Epidemiol., 29(4):260-3, 2001.

Kramer, I. R.; Pindborg, J. J.; Bezroukov, V. \& Infirri, J. S. Guide to epidemiology and diagnosis of oral mucosal diseases and conditions. World Health Organization. Community Dent. Oral Epidemiol., 8(1):1-26, 1980.

Laskin, D. M.; Giglio, J. A. \& Rippert, E. T. Differential diagnosis of tongue lesions. Quintessence Int., 34(5):331-42, 2003.

Marimon, M. \& Garrotela, A. La piel senil. OFFARM, 22(11):80-6, 2003.

Mathew, A. L.; Pai, K. M.; Sholapurkar, A. A. \& Vengal, $\mathrm{M}$. The prevalence of oral mucosal lesions in patients visiting a dental school in Southern India. Indian $J$. Dent. Res., 19(2):99-103, 2008.

Neville, B.; Damm, D. \& Allen, J. Oral \& Maxillofacial Pathology. $2^{\mathrm{a}}$ ed. Philadelphia, Saunders, 2002.

Parlak, A. H.; Koybasi, S.; Yavuz, T.; Yesildal, N.; Anul, H.; Aydogan, I.; Cetinkaya, R. \& Kavak, A. Prevalence of oral lesions in 13- to 16-year-old students in Duzce, Turkey. Oral Dis., 12(6):553-8, 2006.
Petersen, E. P.; Bourgeois, D.; Ogawa, H.; Estupinan-Day, S. \& Ndiaye, C. The global burden of oral diseases and risk to oral health. Bulletin World Health Organ., 83(9):661-9, 2005.

Reichart, P. A. Oral mucosal lesions in a representative cross-sectional study of aging Germans. Community Dent. Oral Epidemiol., 28(5):390-8, 2000.

Ross, M.; Kaye, G. \& Pawlina, W. Histología: Texto y Atlas Color con Biología Celular y Molecular. Buenos Aires, Médica Panamericana, 2006.

Shulman, J. D. An exploration of point, annual, and lifetime prevalence in characterizing recurrent aphthous stomatitis in USA children and youths. J. Oral Pathol. Med., 33(9):558-66, 2004.

Unsal, E.; Paksoy, C.; Soykan, E.; Elhan, A. H. \& Sahin, M. Oral melanin pigmentation related to smoking in a Turkish population. Community Dent. Oral Epidemiol., 29(4):272-7, 2001.

Witkop, C. J. Jr. \& Barros, L. Oral and genetic studies of Chileans, 1960. I. Oral anomalies. Am. J. Phys. Anthropol., 21:15-24, 1963.

\section{Dirección para correspondencia: Araceli Raposo \\ Dpto. de Cirugía bucal \\ Escuela de Odontología \\ Universidad Austral de Chile \\ Valdivia \\ CHILE}

Email: araceli86@gmail.com

Recibido : 13-12-2010

Aceptado: 02-03-2011 